\title{
Original Afrticle \\ Large calculi within malpositioned and malformed kidneys, is percutaneous nephrolithotomy (PCNL) feasible? A Single Center's Experience over 10 Years.
} Awad Ka abneh and Firas Al-Hammouri

\begin{abstract}
:
Background and Purpose: Percutaneous nephrolithotomy (PCNL) for large calculi within malpositioned and malformed kidneys is a challenging problem for urologist because of the abnormal anatomy. The aim of this study was to evaluate and to review our experience with PCNL in malpositioned and malformed kidneys with large calculi.

Patients and Methods: Between January 2000 and December 2009, we performed PCNL in 36 patients with large calculi in malpositioned and malformed kidneys, including 16 patients with horseshoe kidneys, 11 patients with rotational anomalous, three patients with transplanted kidney and six patients with ectopic pelvic kidneys.

After appropriate preoperative evaluation, the procedure was performed by choosing anterior, posterior or laparoscopic assisted approaches under fluoroscopic and ultrasound guidance. PCNL access was made in the upper pole of the kidney in $21(58.3 \%)$ patients, in the midpole in 14 (38.9\%) patients, and in the lower pole in one patient $(2.8 \%)$.

Results: The mean age of the patients was 37.5 years (range 21- 57) with male: female ratio 2.7: 1 , the mean stone size was $2.9 \mathrm{~cm}$ (range 2.1-4.9). Complete stone removal was achieved in 26 $(72.2 \%)$ patients. A second-look procedure for residual stone removal was required in nine patients $(25 \%)$, five of them via the same tract and three patients required another access, four of them became stone free, four patients required adjuvant ESWL sessions for the residual stones, and one patient converted to open surgery due to difficulty in creating an access.

The mean operating time for PCNL was 95 minutes (range 45-120), and the mean hospital stay was 3.7 days (range 3-8 days). Blood transfusion was required for three (8.3\%) patients, transient postoperative pyrexia encountered in six $(16.7 \%)$ patients and one patient had persistent urine leak $(2.8 \%)$. Serious complications were encountered, small bowel perforation was occurred in two $(5.6 \%)$ patients, and both were treated by laparatomy with primary repair of injury with uneventful outcome.

Conclusion: Patients with malposition and malformed kidneys and large calculi can be managed safely and effectively with PCNL when they are properly selected and appropriately assessed before operation. Stone management in malformed and malposition kidneys is challenging, and establishing percutaneous renal access is the most crucial step in the procedure.
\end{abstract}

Keywords: laparatomy, pyelolithotomy, laparoscopic.

$\mathrm{F}$ usion and rotation anomalies are among the most common anomalies of the kidneys especially the horseshoe kidneys that have abnormal mixtures of malrotation, ectopia and fusion ${ }^{1}$. Fusion of the lower poles prevent normal ascent of the kidneys causes malrotation of the collecting

Prince Hussein Bin Abdullah Urology Center, King Hussein Urology Center, Amman Jordan Correspondence address: Firas Al-Hammouri . P.O.Box: 411, Amman 11953, Jordan Firas_hammouri@yahoo.com system in addition to the high insertion of the ureter that is associated with significant rate of ureteropelvic obstruction ${ }^{2}$. Renal stones are the most common complication in malformed kidneys due to stasis and impaired drainage, calculi in horseshoe and ectopic kidneys present unique challenges in decision-making and technical aspects of treatment ${ }^{2}$. In the modern era almost all renal stones can be treated with minimal invasive or non-invasive techniques. Percutaneous nephrolithotomy (PCNL) for calculi within malformed and 
malposition kidneys can be challenging because of the abnormal anatomy and the required technical modification ${ }^{2}$. We present our experience in management of renal calculi in malformed and malposition kidneys by PCNL.

\section{Patients and Methods}

Between January 2000 and December 2009, we performed PCNL procedure on 36 patients with calculi in malposition and malformed kidneys, including 16 patients with horseshoe kidneys, 11 patients with rotational anomalous, six patients with ectopic pelvic kidneys and three patients with kidney transplantation.

Patient's pre-operative assessment included medical history, physical examination, urine analysis, urine culture, serum hematocrit, kidney function test, renal ultrasonography and renal CT scan (stone protocol).

Each case had a documented negative urine culture or treated with antibiotic according to the culture sensitivity. The procedure was done under general anesthesia with prophylactic antibiotics using second generation cephalosporin. In dorsal lithotomy position a retrograde catheter was inserted into the proper site and connected to a syringe containing contrast media. The position then was changed to prone, supine, or lateral position according to the access. All patients with horseshoe kidneys were approached in prone position except in two cases where we used the technique of laparoscopic assisted approach in lateral extra-peritoneal position. In patients with malrotated kidneys the approach was prone position. In all patients with ectopic kidneys the approach was semisupine $45^{\circ}$ position with pillow underneath the working site of the abdomen, and supine position in cases of kidney transplant.

The procedure was started by a retrograde pyelogram via the previously inserted ureteric catheter using contrast material. Percutaneous renal access was established under fluoroscopic guidance with help of ultrasound in cases of pelvic kidneys. The initial puncture was done using chipa needle (18G) followed by floppy tip hydrophilic guide wire
$(0.0032 \mathrm{G})$. Before starting dilatation of the access in patients with pelvic and transplanted kidneys, we injected antigrade contrast material while the needle was withdrawn to detect any bowel or vascular injuries. The dilatation was started using facial dilators from $6 \mathrm{~F}$ to $12 \mathrm{~F}$ followed by balloon dilatation of the tract and insertion of Amplatz sheath size 30F over Amplatz dilator. In some cases according to its availability at our center, one step nephromax was used. A 26F nephroscope was introduced through the sheath, with help of isotonic saline for irrigation and clear visualization. Fragmentation of the visualized stones by pneumatic lithoclast was done followed by removal of pieces using stone grasping forceps and suction. The completeness of stone clearance was achieved by fluoroscopy at the end of the procedure, and a Foley catheter size $20 \mathrm{~F}$ was inserted in all cases as a draining nephrostomy and kept for 24-72 hours post-operatively.

Percutaneous access was obtained via a single tract through the upper pole calyx in nine patients with horseshoe kidneys $(56.3 \%)$, in three patients with malrotated kidneys $(27.3 \%)$, in six patients with pelvic ectopic kidneys (100\%), and in the three patients with kidney transplant $(100 \%)$. The access was done through the middle pole calyx in seven patients with horseshoe kidneys (43.7\%), and in seven patients with malrotated kidneys $(63.6 \%)$. In one patient with malrotated kidney the access was established through the lower pole calyx $(9.1 \%)$.

All our patients were kept on intravenous second generation cephalosporin for 48 hours. Retrograde and Foley catheters were removed in the first post-operative day. Nephrostomy tubogram was done for all patients 48 hours after surgery to detect any significant residual stones or pelvi-calyceal system injury and to document the free drainage to the urinary bladder. A second look PCNL procedure or adjuvant ESWL were decided according to the significance and accessibility of residual stones. The second look procedure was either through the same previously created tract or through a new access. JJ stent was considered 
if persistent urine leak occurred after removal of nephrostomy tube.

KUB and ultrasound were done for all of our patients six weeks after surgery.

\section{Results}

Thirty six patients with stones in malposition and malformed kidneys were treated by PCNL. The mean age of the patients was 37.5 years (range 21-57), with male to female ratio of $2.7: 1$, the average stone size was $2.9 \mathrm{~cm}$ (range $2.1-4.9 \mathrm{~cm}$ ), the left to right ratio was 2.7:1, and all patients with ectopic pelvic kidneys had their kidneys on the left side.

Complete stone removal was achieved in 26 $(72.2 \%)$ patients, a second-look procedure was needed in nine (25\%) patients for significant residual sizable stones that were not removed by the first session because of presence of significant bleeding that obscure the view or migratation of the stone to inaccessible calyx. For these we used the same tract in five patients and achieved stone clearance in three of them, thus increasing the stone free clearance rate to $(80.6 \%)$. We create another tract through a different calyx to remove residual stone in three patients, and one of them became stone free but the other two still they harbor residual stone. Adjuvant ESWL was performed to four patients with residual stones and became stone free after two to three sessions with two weeks interval between the sessions. One patient with horseshoe kidney required open pyelolithotomy due to difficulty in creating an access to the kidney.

The mean operative time for PCNL was 95 minutes (range 45-120 minutes), this time including the time for insertion of retrograde catheter, the mean operative time for a second look procedure was 48 minutes (range 28-95 minutes). The average hospital stay was 3.7 days (range 3-8 days). The mean drop in hemoglobin was $2.8 \mathrm{gm} / \mathrm{dl}$ (range 0.9-5.1 $\mathrm{mg} / \mathrm{dl}$ ), three patients required two units of previously crossed matched blood $(8.3 \%)$. Six patients developed transient pyrexia in the first 24 hours (16.7\%), the fever subsided by antipyretics and hydration in addition to the scheduled antibiotics that were already started.

One of our patient developed persistent urine leak after removal of nephrostomy tube on the third post-operative day (2.8\%). Cystoscopy and retrograde pyelogram performed and showed small lower ureteric stone, that was removed using rigid ureteroscope followed by insertion of $\mathrm{JJ}$ stent and the patient was discharge the second day.

Serious complications were encountered in our series of patients. Small bowel perforation was discovered during laparotomy two hours after the PCNL procedure for a patient with a stone in a transplanted kidney. Due to significant intra-peritoneal fluid collection, primary closure of the distal ilium lacerated wound was done with normal post-operative recovery. Another patient with pelvic kidney had also distal small bowel injury, but was detected after removal of nephrostomy tube on the third post-operative day by leakage of a small bowel content through the nephrostomy tube site. Urgent laparotomy with primary closure was also done with smooth post-operative recovery. Table (1) summarizes the results.

The stone composition in our patients was mixed type in $25(69.4 \%)$ patients $(70 \%$ calcium oxalate monohydrate and $30 \%$ calcium oxalate dehydrate), cysteine stone in five $(13.9 \%)$ patients, MAP (magnesium ammonium phosphate) stones in three $(8.3 \%)$ patients and uric acids stones in three $(8.3 \%)$ patients.

\section{Discussion}

The endoscopic treatment of large renal stones still remains a challenge, and this becomes extreme when it is in malpositioned kidneys. PCNL in anomalous kidneys appears to be of great concern due to multiple factors such as abnormal renal and calyceal orientation, abnormal relations of the calices to the renal pelvis and upper ureter, relative renal immobility interfering with maneuverability of the equipment, abnormal renal relations with other intra abdominal organs, and abnormal dispensation of renal vasculature $^{3}$. 
Table (2): Results of the PCNL procedure for malposition and malformed kidneys

Mean operative time (Range)

Mean operative time $2^{\text {nd }}$ look (Range)

2nd look through same tract

2nd look through different access

Stone clearance with PCNL monotherapy

Stone clearance rate after second look

Mean hospital stay (Range)

Mean drop in $\mathrm{Hb}$ (Range)

Blood transfusion

Duration of nephrostomy in PCNL monotherapy

Transient postoperative pyrexia

Postoperative urine leak

Small bowel perforation

The experience with minimally invasive treatment of large stones in pelvic ectopic kidneys is limited to some case reports and small series. The various approaches have included pure laparoscopic pyelolithotomy, laparoscopy assisted PCNL, and ultrasoundguided PCNL. These are attractive modalities because they have the advantages of reduced analgesic requirement, shorter hospital stay, early convalescence, and better cosmoses, in addition to excellent safety and efficacy profiles ${ }^{4}$.

Patients with large-volume calculi located within malpositioned kidney present a unique challenge to the endourologist. Although ESWL is commonly applied to calculi residing in orthotopic kidneys, it can be challenging to apply to malpositioned kidneys, because positioning the stone at the focal point of the shockwave can be difficult or even impossible. Nevertheless, several groups have reported their experiences with ESWL for patients harboring calculi in ectopic kidneys ${ }^{5,6}$.

Despite the widespread appeal of PCNL in the management of large-volume renal stones, its application in patients with malpositioned kidney calculi is one of the more challenging endourologic procedures, requiring the surgeon to strive for a balance between complete stone clearances with acceptable patient morbidity.

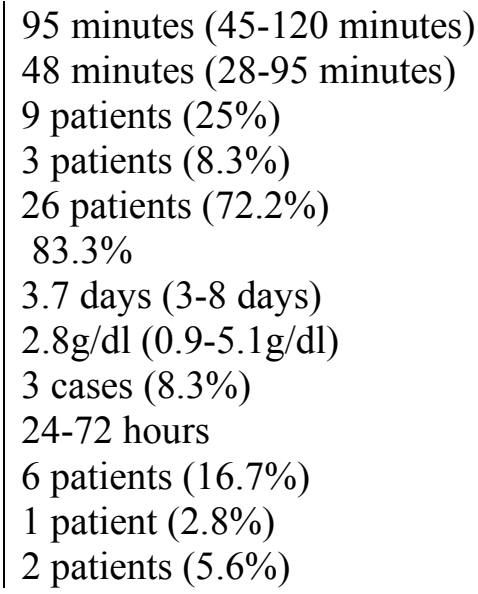

In our current series, because of the large stone burdens in all patients (average stone size of $2.9 \mathrm{~cm}$ ); ESWL was not an appropriate treatment option for our patients.

Weizer and associates reported in a retrospective study, that patients with renal calculi in anomalous kidneys can undergo ureteroscopy ${ }^{7}$. Although ureteroscopy is a reasonable option for select patients harboring calculi in pelvic kidneys, we believe that the average stone burden in our current series was $2.9 \mathrm{~cm}$ making this approach (ureteroscopy) more difficult with low stone free clearance rate.

Although Raj et al. ${ }^{8}$ claimed use of flexible nephroscope in $80 \%$ patients to access stones in various calices adequately in horseshoe kidneys, we are using flexible nephroscope for diagnosis of not accessible stones by rigid nerphroscope but at our institute we are lacking the feasibility of laser stone management so it is difficult to draw a comparison.

Complete stone clearance can sometimes be difficult to assess in malposition kidneys especially in ectopic pelvic kidneys by plain film or by using fluoroscopy intra-operatively due to overlying bones, and that's why some authors advice a second look procedure in these patients 9 . We did a second look procedure for nine patients due to residual stones, none of them for pelvic or transplanted kidneys. 
PCNL remains an operation with potential significant morbidity and necessitates thoughtful planning considerations. Hemorrhage resulting in blood transfusion, renal artery embolization, or need for an emergent exploration with high possibility of nephrectomy remain a concern but are uncommon. Earlier experiences reported the need for blood transfusion in up to $34 \%$ of patients undergoing PCNL ${ }^{10-12}$. The blood transfusion rates reported in the literature specifically for PCNL of staghorn stones vary from 14 to $24 \%$, with the variability likely a reflection of differing surgical techniques, diverse patient populations, stricter indications for transfusion overall, and the individual surgeon's reflex to transfuse ${ }^{13-15}$. Our experience reveals that no additional risk of hemorrhage is associated with PCNL in anomalous kidneys in contrast to normal kidneys; our blood transfusion incidence was $(8.3 \%)$.

The incidence of bowel injury is increased because of the presence of adhesions between the bowel and the peritoneal surface of kidney, which has been reported at 13\%-27\% in different series ${ }^{16,17}$. We had two cases of small bowel injury $(5.6 \%)$, they occurred during our first case of PCNL for pelvic kidney, and in PCNL for a kidney transplant for obese female patient that had overlying undetected small bowel that was missed even with the use of antigrade contrast injection during withdrawal of the chipa needle.

\section{Conclusion}

Patients with malpositioned or malformed kidneys and large calculi can be managed safely and effectively with PCNL when they are properly selected and appropriately assessed before operation. Stone management in malformed and malpositioned kidneys is challenging and establishing percutaneous renal access is the most crucial step in the procedure.

\section{References:}

1. Lojanapiwat B. Perctaneous nephrolithotomy (PCNL) in kidneys with fusion and rotation anomalies. J Med Assoc Thai 2005; 88: 1426-9.
2. Stein RJ, Desai MM. Management of urolithiasis in the congenitally abnormal kidney (horseshoe and ectopic). Curr Opin Urol 2007; 17: 125-31.

3. Rana AM, Bhojwani JP, percutaneous nephrolithotomy in renal anomalies of fusion, ectopia, rotation, hypoplasia, and pelvicalyceal aberration: uniformity in heterogeneity. J Endourol. 2009 Apr;23(4):609-14

4. Hamdy A, El-kappany MD, El-nahas AR, et al. combination of laparoscopy and nephroscopy for treatment of stones in pelvic ectopic kidneys. journal of endourology 2007; 21(10): 1131-1135.

5. Tunc L, Tokgoz H, Tan MO, et al. Stones in anomalous kidneys: results of treatment by shock wave lithotripsy in 150 patients. Int J Urol 2004; 11: 831836.

6. Talic RF: Extracorporeal shock-wave lithotripsy monotherapy in renal pelvic ectopia. Urology 1996; 48: 857-861.

7. Weizer AZ, Springhart WP, Ekeruo WO, et al. Ureteroscopic management of renal calculi in anomalous kidneys. Urology 2005; 65: 265-269.

8. Raj GV, Auge BK, Weizer AZ, et al. Percutaneous management of calculi within horseshoe kidneys. J Urol 2003; 170: 48-51.

9. Desai MR, Jasani A. Percutaneous nephrolithotripsy in ectopic kidneys. Journal of Endourology 2000; 14: 289-292.

10. Eshghi AM, Roth JS, and Smith AD: Percutaneous transperitoneal approach to a pelvic kidney for endourological removal of staghorn calculus. J Urol 1985; 134: 525-527.

11. Figge M: Percutaneous transperitoneal nephrolithotomy. Eur Urol 1988; 14: 414-416.

12. Toth C, Holman EE, Pasztor I, et al. Laparoscopically controlled and assisted percutaneous transperitoneal nephrolithotomy in a pelvic dystopic kidney. J Endourol 1993; 7: 303-305.

13. Stoller ML, Wolf JS Jr, St Lezin MA. Estimated blood loss and transfusion rates associated with percutaneous nephrolithotomy. J. Urol 1994; 152:1977-1981.

14. Lang EK. Percutaneous nephrostolithotomy and lithotripsy: A multi-institutional survey of complications. Radiology 1987; 162: 25-30.

15. Clayman RV, Surya V, Hunter D, et al. Renal vascular complications associated with the percutaneous removal of renal calculi. J Urol 1984; 132: 228-230.

16. Holman E, Toth C. Laparoscopically assisted percutaneous transperitoneal nephrolithotomy in pelvic dystopic kidneys: experience in 15 successful cases. $J$ Laparoendosc Adv Surg Tech A. 1998; 8: 431-435. 17. El-Kappany HA, El-Nahas AR, Shoma AM, et al. Combination of laparoscopy and nephroscopy for treatment of stones in pelvic ectopic kidneys. J Endourol 2007; 21:1131-1135. 